\title{
FORUM
}

\section{Collision and the Perspectivity}

\author{
Frank Coffman Bell
}

Captain W. Burger and Captain A. G. Corbet in their general comment ${ }^{1}$ on the meeting of the Technical Committee in London, 16 April 1969, gave a curious and instructive hypothetical example of end-on encounter, a problem in navigation which they and others have found by experiments to have importance and practical difficulties. Their example is set in surface navigation at sea, and especially when using radar in fog. Two ships close with each other (in their example head-on and green-to-green), the two tracks being straight and parallel, and separated (in their example by half a mile). Should both ships stand on ? My answer is affirmative, my argument difficult.

If one makes the fruitful assumption that neither ship in this example alters course or speed, and then tries to plot The Edge ${ }^{2}$ [ of regression of The (mutual) Line (of bearing)], he discovers that the result is not a non-singular parabola, but rather simply a fixed finite point, i.e. at best a degenerate parabola-yet clearly there can be no finite collision, and collision at infinity is an empty threat. What do plotters of The Edge make of this paradox ? Here handbook summaries will not do.

To invite connecting projective geometry and the theory of collision and its avoidance, I quote Professor Henrici, ${ }^{3}$ passim, interpolating my own additional words and comments in brackets :

'If the points at infinity in two projective rows correspond . . . corresponding segments are proportional . . . [and] we call the rows similar.' [If corresponding segments are not just proportional, but equal, I will use the term equal rather than similar.]

'Theorem.- Two projective rows are similar if to the point at infinity in one corresponds the point at infinity in the other; and conversely, if two rows are similar [then] they are projective, and the [two] points at infinity are corresponding points.

'From this the [two] well-known propositions follow:

[I] 'Two [non-parallel] lines are cut proportionally by a series of parallels. The [resulting] rows [in the two non-parallel lines] are perspective, with centre of projection [of their perspectivity] at infinity.' [This is the geometric basis of finite collision when The Line ceases to rotate and its successive positions form a series of parallels. If the 'two lines' are parallel, however, then there is no finite collision.] 
[II] 'If two similar [but not equal] rows are placed parallel, then the lines joining homologous points pass through a common [fixed finite] point.' [-the fixed finite point centre of projection of the perspectivity. This is the geometric basis of a miss when the tracks are parallel and distinct, The Line having identically one fixed finite point centre of rotation. If the 'two rows' are equal, however, then there is no finite collision.]

'Any two [of all the ] tangents to a [given non-singular] parabola are cut by all [the] others in projective rows; but the line at infinity being one of the tangents [to any non-singular parabola], the points at infinity in the [above two projective] rows are corresponding points, and the [above two projective] rows [are] therefore similar. Hence the theoremThe tangents to a Inon-singular] parabola cut each other proportionally.' [It is this property of the non-singular parabola which makes possible the identification of, and plotting The Edge as a definite non-singular curve in, a finite crossing situation free from real threat of collision.]

Using the propositions [I] and [II] given above, two ships can eliminate the ambiguous cases of collision at infinity, and then identify the case where The Edge degenerates into a finite fixed point as a miss by parallel and distinct tracks. Such a procedure has a sort of parity with the one they commonly use to identify the apparently better known case, viz., that where the successive positions of The Line in a finite crossing situation form a series of parallels, as a finite collision. But, of course, the centre of projection in any perspectivity returns no radar echo or reflection, and can be found in all generality only by 'plotting' .

The general statement, 2 'that a miss will certainly occur if two ships maintain course and speed while The Line's rotation does not vanish', seems not to be defeated by the parallel miss, although in the reference given it was not proved for any singular case. The extenuating remark can be made that there exists a class of cases (equal rows) in which, although The Lines form a series of parallels, there is no finite collision. These last are the cases where two ships are on parallel and distinct tracks at constant and equal courses and speeds. Hence it seems that the negative form used in the above given general statement is slightly stronger than the more usual positive form reading 'hit' for 'miss', and 'does vanish' for 'does not vanish'.

\section{REFEREN CES}

1 Burger, W. and Corbet, A. G. (1969). General Comment in The Revision of the Collision Regulations. This Journal, 22, 306.

2 F. C. Bell (1969). The Edge of a Needless Collision (Forum). This Journal, 22, 513.

3 Professor Olaus Magnus Friedrich Henrici, Ph.D., LL.D., F.R.S. (1910). Geometry II. Projective Geometry, Encyclopaedia Britannica, Eleventh Edition, Vol. II, 694, 699, 707. 